\title{
Correlation of Transferrin Saturation and Serum Ferritin with Bone Mass Density in Adult Transfusion Dependent Beta-Thalassemia Patients
}

\author{
Tubagus Djumhana \\ Atmakusuma (iD) 1,2 \\ Jeffry Beta Tenggara ${ }^{2,3}$ \\ 'Division of Hematology-Medical \\ Oncology, Department of Interna \\ Medicine, Dr. Cipto Mangunkusumo \\ General Hospital, Jakarta, Indonesia; \\ ${ }^{2}$ Faculty of Medicine Universitas \\ Indonesia, Jakarta, Indonesia; ${ }^{3}$ Division of \\ Hematology-Medical Oncology, \\ Department of Internal Medicine, \\ MRCCC Siloam Hospital Jakarta, Jakarta, \\ Indonesia
}

Correspondence: Tubagus Djumhana Atmakusuma

Division of Hematology-Medical

Oncology, Department of Interna

Medicine, Dr. Cipto Mangunkusumo

General Hospital, Jalan Diponegoro No.

7I, Jakarta Pusat, DKI, Jakarta, Indonesia

Email hom_fkui@yahoo.com
Background: The use of regular blood transfusions and iron chelation therapy to treat thalassemia has improved survival and increased the incidence of osteoporosis. Moreover, iron toxicity is one of the contributing factors that reduce bone mass density in adult transfusion-dependent beta-thalassemia patients. Therefore, this study aims to determine the proportion of low bone mass density in adult thalassemia patients and transferrin saturation, as well as serum ferritin, which correlates to the skeletal condition.

Methods: This is a cross-sectional study conducted in Thalassemia and Hematology Medical Oncology Clinics of Cipto Mangunkusumo Hospital in March 2016. The anthropometric data and hemoglobin levels were obtained before transfusion. Subsequently, the average ferritin levels, bone mineral density, and radiographic results were obtained.

Results: The percentage of adult thalassemia major and intermedia patients with low bone mass density was $68 \%$. Also, there was a weak inverse correlation between bone mass density and transferrin saturation $(\mathrm{r}=-0.329, \mathrm{p}=0.01)$, while no correlation was shown between bone mass density and ferritin $(r=-0.088, \mathrm{p}=0.504)$. The transferrin saturation cutoff point value used to distinguish the incidence of low and normal bone density in patients with transfusion-dependent beta-thalassemia was $89.5 \%$. In addition, there was weak correlation between Singh index and bone mass density $(r=0.273, p=0.038)$.

Conclusion: Among the transfusion-dependent beta-thalassemia patients, $68 \%$ had low bone mass density, which inversely correlated to transferrin saturation. Furthermore, the cutoff value of transferrin saturation to differentiate the incidence of low and normal bone density in thalassemia major compared to thalassemia intermedia was $89.5 \%$. Singh Index correlates weakly with bone mass density and might be used to detect low bone mass density in remote healthcare facilities.

Keywords: bone mass density, ferritin, thalassemia, transfusion dependent beta, transferrin

\section{Background}

Thalassemia is a group of hereditary disorders associated with the defective synthesis of alpha or beta-globin, which are inherited as pathologic alleles of the globin genes on the chromosomes $11(\beta)$ and $16(\alpha) .{ }^{1}$ The damage of the membrane structure leads to accelerated apoptosis and premature destruction of the erythroid precursors in the bone marrow (ineffective erythropoiesis). ${ }^{2}$ Meanwhile, ineffective erythropoiesis reduces bone mass density and increases the incidence of fractures. ${ }^{3}$

The provision of regular blood transfusions has been shown to increase the survival of patients with thalassemia; however, it leads to the occurrence of iron 
overload, which progresses to iron toxicity. The iron toxicity is mediated through non-transferrin bound iron (NTBI), a free radical, which is among the factors that contribute to organ damage in thalassemia. ${ }^{4,5}$ Since NTBI levels are measured only at several research centers abroad, therefore, to measure iron toxicity, surrogate markers such as saturation transferrin and serum ferritin are used. $^{6}$

Iron toxicity functions as one of the contributing factors to bone density reduction in adult patients with thalassemia. Moreover, ineffective erythropoiesis causes expansion of the bone marrow and leads to mechanical disruption of the bone structure. ${ }^{7}$ Currently, there have not been studies on the correlation between transferrin saturation and ferritin parameters with bone density or NTBI receptors in bone.

The use of transfusion and iron chelation therapy to treat thalassemia patients has improved survival; however, regular transfusions cause an increase in the incidence of osteoporosis. $^{8-11}$ Out of the population of patients with thalassemia that have received adequate transfusion therapy, the incidence of osteoporosis reported was approximately $66 \% .^{12}$ A previous study by Hashemieh et $\mathrm{al}^{13}$ reported a $23 \%$ increased incidence of osteopenia in thalassemia major and $8 \%$ in thalassemia intermedia. ${ }^{13}$ Furthermore, a study also stated that incidence of osteoporosis is increased by $60 \%$ in thalassemia major and intermedia are $60 \%$ and $81.6 \%<$, respectively. ${ }^{13}$ Meanwhile, bone disorder as a complication of thalassemia causes negative psychological impact, lowers the quality of life, and increases treatment costs.

In Indonesia, the incidence of osteoporosis/low bone density is between $28 \%$ and $32 \%{ }^{14}$ The pathogenesis of reduced bone density in thalassemia is associated with the expansion of bone marrow caused by ineffective erythropoiesis, sexual maturation disorders, hormonal disorders such as hypothyroidism, hypoparathyroidism, diabetes mellitus, and direct iron toxicity on the bone. Furthermore, the use of desferrioxamine iron chelation therapy also plays an important role in the incidence of low bone density. ${ }^{15}$

The measurement of bone mass dual X-ray absorptiometry (DXA) in the lumbar area, femoral neck, and forearm is a non-invasive examination that accurately assesses bone density, ${ }^{16}$ however, these tests are not widely available in Indonesia. To overcome this limitation, a simple Singh Index measurement using a conventional X-ray modality is considered. Although this method is widely available, studies on the evaluation of bone density in patients with thalassemia had not been examined, especially in Asian population. Therefore, this study aims to determine the proportion of low bone mass density in adult thalassemia patients and the correlation between transferrin saturation and serum ferritin with the skeletal condition.

\section{Methods}

This is a cross-sectional study conducted in Thalassemia together with Hematology and Medical Oncology Clinics of Cipto Mangunkusumo Hospital in March 2016. The inclusion criteria were patients above 18 years, which were diagnosed with thalassemia based on HighPerformance Liquid Chromatography (HPLC) or microcapillary, received a regular red blood transfusion at least once a month, and agreed to participate in the study. Subsequently, blood samples, inspection dual X-ray absorptiometry (DXA), and bone radiographs were acquired, while $\mathrm{GE}^{\circledR}$ Lunar iDXA ${ }^{\mathrm{TM}}$ system was used to measure bone densitometry. In this study, the exclusion criteria were patients that refused to participate, use drugs that affect bone density such as corticosteroids, and are with clinical signs of infection such as fever.

The data collected included name, age, complete address, phone number, weight, height, puberty status, hemoglobin level before transfusion, average ferritin levels during the last 6 months to 1 year, bone density measurement with DXA instruments, and bone radiographic examination.

The data obtained were analyzed using IBM ${ }^{\text {TM }}$ SPSS Statistics $^{\circledR} 20$ and Statistical correlation tests were carried out using non-parametric Spearman test. The Z-score from bone densitometry was analyzed as a continuous variable that correlated with the serum ferritin, transferrin, and femoral Singh index. Dichotomous bone mass density data were only used to determine the percentage of patients with low bone density.

This study was conducted in line with the Declaration of Helsinki and was approved by the Ethical Committee of the Faculty of Medicine, Universitas Indonesia. Also, appropriate informed consent was from all subjects.

\section{Results}

\section{Demographic Data}

The data were from 60 patients, which consisted of 27 males $(45 \%)$ and 33 females (55\%). The median age was 
25 years, which ranged from 18 to 68 years as shown in Table 1. Meanwhile, the median level of ferritin was 3881 $\mathrm{ng} / \mathrm{mL}$ with the lowest value of $645 \mathrm{ng} / \mathrm{mL}$ and the highest value of $15,437 \mathrm{ng} / \mathrm{mL}$. The median transferrin saturation was $86 \%$ with the lowest value of $20 \%$ and the highest value of $120 \%$. These results showed that the median bone mass density values were -1.1 with the lowest value of -5.7 and the highest of 2.6. (Table 1).

\section{The Proportion of Low Bone Mass Density}

Bone mass density was evaluated using the Dual X-ray Absorptiometry (DXA). The results showed that 41 (68\%) patients had low bone mass density $(Z$ score $<-2)$, while 19 (32\%) had normal bone density ( $Z$ score $\geq 2)$.

\section{The Correlation Between the Saturation of Transferrin or Ferritin in Bone Density}

The transferrin saturation, ferritin, and bone mass density of 60 patients were examined with the KolmogorovSmirnov test for normality, which obtained a probability value of $0.000(p<0.05)$; therefore, non-parametric tests were used. Spearman correlation test was used to determine the correlation between transferrin saturation and ferritin with the bone mass density.

There was a weak inverse correlation between transferrin saturation and bone mass density $(\mathrm{r}=-0.329$, $\mathrm{p}=0.01$ ), while there was no significant correlation between the levels of serum ferritin with bone mass density $(r=-0.088, p=0.504)$ (Table 2$)$.

\section{The Cut-Off Point of Transferrin Saturation Associated with Low Bone Density}

Curve analysis by the receiver operating curve (ROC) was generated to determine the portion of patients predicted to have a low bone mass density in adult thalassemia major and intermedia by the transferrin saturation values. Figure 1 showed a ROC curve value of transferrin saturation 0.727 , p-value 0.005 , and $95 \%$ CI $0.595-0.858$, which indicated a good value. Moreover, the cut-off point to determine transferrin saturation to distinguish low and normal bone densities in adult patients with thalassemia major and intermedia was analyzed. Based on the result, a transferrin saturation value of $89.5 \%$ was obtained as the most excellent point (Figure 2).
Table I General Characteristics of Research Subjects

\begin{tabular}{|l|l|}
\hline General Characteristics & $\mathbf{N}=6 \mathbf{6 0}$ \\
\hline Gender, $\mathrm{n}(\%)$ & \\
Male & $27(45 \%)$ \\
Female & $33(55 \%)$ \\
\hline Classification of Thalassemia & \\
Thalassemia major (TM) & $19(32 \%)$ \\
Thalassemia intermedia (TI) & $4 \mathrm{I}(68 \%)$ \\
\hline Classification of thalassemia & \\
Thalassemia Beta Thalassemia & $26(43 \%)$ \\
Beta/HBE & $34(57 \%)$ \\
\hline Iron status & \\
Ferritin (ng/mL), median (min-max) & $3881(645-15,437)$ \\
Transferrin saturation, median (min-max) & $86(20-120)$ \\
\hline Anthropometry & \\
Weight (kg), median (min-max) & $43(25-85)$ \\
Height (cm), median (min-max) & $154(123-170)$ \\
Short stature, N (\%) & $28(47 \%)$ \\
Facies Cooley, N (\%) & $32(53 \%)$ \\
BMD Z score, median (min-max) & $-1.1(-5.7$ to 2.6) \\
\hline Laboratory values & \\
Pre-transfusion hemoglobin (mg/dL), mean (SD) & $8.08(1.09)$ \\
hsCRP (mg/L), mean (SD) & $3.15(0.34-4.4)$ \\
Magnesium (mEq/L), mean (SD) & $2.02(0.24)$ \\
Sodium (mEq/L), mean (SD) & $137.38(4.30)$ \\
Potassium (mEq/L), mean (SD) & $4.06(0.42)$ \\
Chloride (mEq/L), median (min-max) & $99(89-114)$ \\
Calcium (mg/dL), mean (SD) & $8.91(0.49)$ \\
Phosphate (mg/dL), median (min-max) & $4(2.2-6.20)$ \\
Transferrin saturation (\%), median (min-max) & $86(20-112)$ \\
Serum ferritin (ng/mL), median (min-max) & $3881(645-15,437)$ \\
\hline Femoral Singh Index, median (min-max) & $5(1-6)$ \\
\hline
\end{tabular}

Abbreviations: BMD, bone mass density; SD, standard Ddeviation; hsCRP, highsensitive C-reactive protein.

\section{Cut Point (Cut-Off) of Serum Ferritin Associated with Low Bone Density in Adult Thalassemia Patients}

Based on previous analysis (Table 2), there was no significant correlation between ferritin and bone density, and can not be determined as cut-off point ferritin value to

Table 2 Correlation of Transferrin Saturation, Serum Ferritin and Singh Index with Bone Mass Density

\begin{tabular}{|l|l|l|}
\hline Variables & $\mathbf{r}$ & $\mathbf{p}^{*}$ \\
\hline Transferrin saturation - BMD & -0.329 & 0.010 \\
Serum Ferritin - BMD & -0.088 & 0.504 \\
Femoral Singh Index - BMD & 0.273 & 0.038 \\
\hline
\end{tabular}

Note: *Spearman correlation test.

Abbreviation: BMD, bone mass density. 


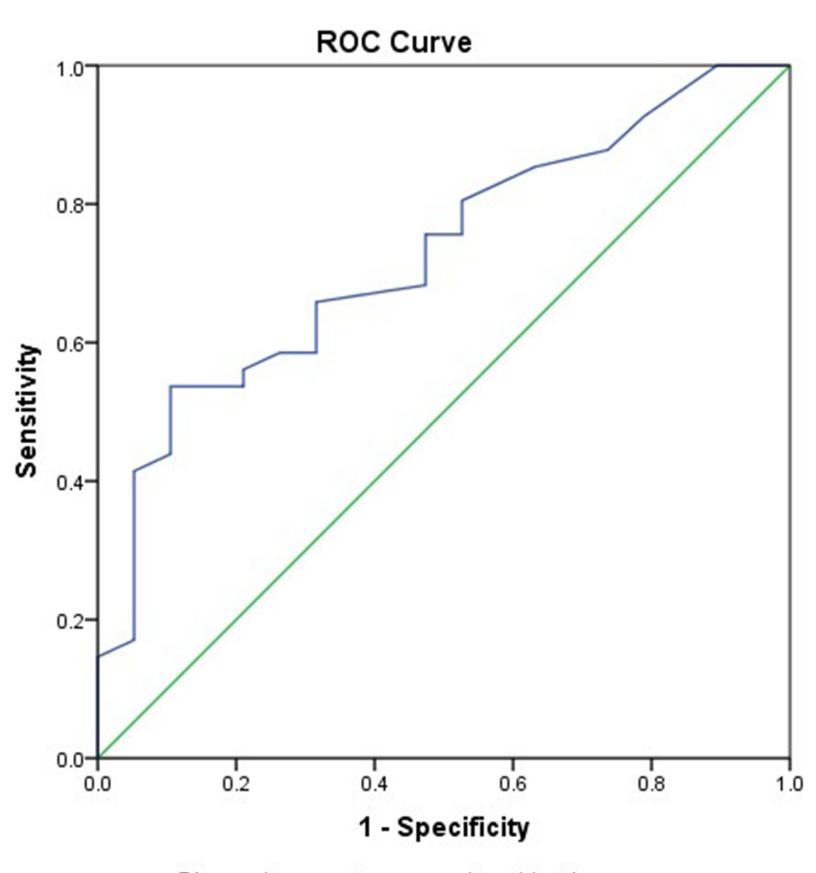

Diagonal segments are produced by ties.

Figure I Transferrin saturation values receiver operating characteristic (ROC) curve for prediction of future occurrence of low bone density in adult thalassemia patients.

distinguish low and normal bone density in patients with thalassemia major and intermedia adults.

\section{The Correlation Between Bone Density Values with Singh Index in Thalassemia Adult Patients}

The Singh index value and the bone mass density in 60 patients were examined based on the Kolmogorov-
Smirnov test to obtain a normality probability value of 0.000 ( $\mathrm{p}<0.05)$. Furthermore, the Spearman correlation test was conducted to evaluate the correlation between Singh index and bone mass density. The results showed that there was weak correlation (Table 2) between Singh Index and bone mass density $(r=0.273, p$-value $=0.038)$.

\section{Discussion}

The wide range of serum ferritin and transferrin saturation values occurred due to the differences in patients' compliance with iron chelation therapy. The amount of blood transfusion should not be a confounding factor since the subjects receive regular blood transfusion every month. In this study, the ferritin value used was obtained by calculating the average ferritin values through several examinations in the previous year. Since the value can be influenced by many factors besides the iron content in the blood such as inflammation and hepatic impairment, averaging several measurements minimized that concern.

There were $68.3 \%$ subjects with low bone density. This value is higher than the incidence of low bone mass density in the general population $(23 \%),{ }^{14}$ In addition, $47 \%$ of the patients had short stature. Increased incidence of low bone density and osteoporosis in patients with thalassemia was also reported by Hashemieh et $\mathrm{al}^{10}$ in Iran $(65 \%)$ and Merchant et $\mathrm{al}^{17}$ in India $(81 \%)$. Based on the similarity of our results with other studies, low bone mass density is considered a frequent complication in patients with thalassemia, which requires early diagnosis and prompt management.

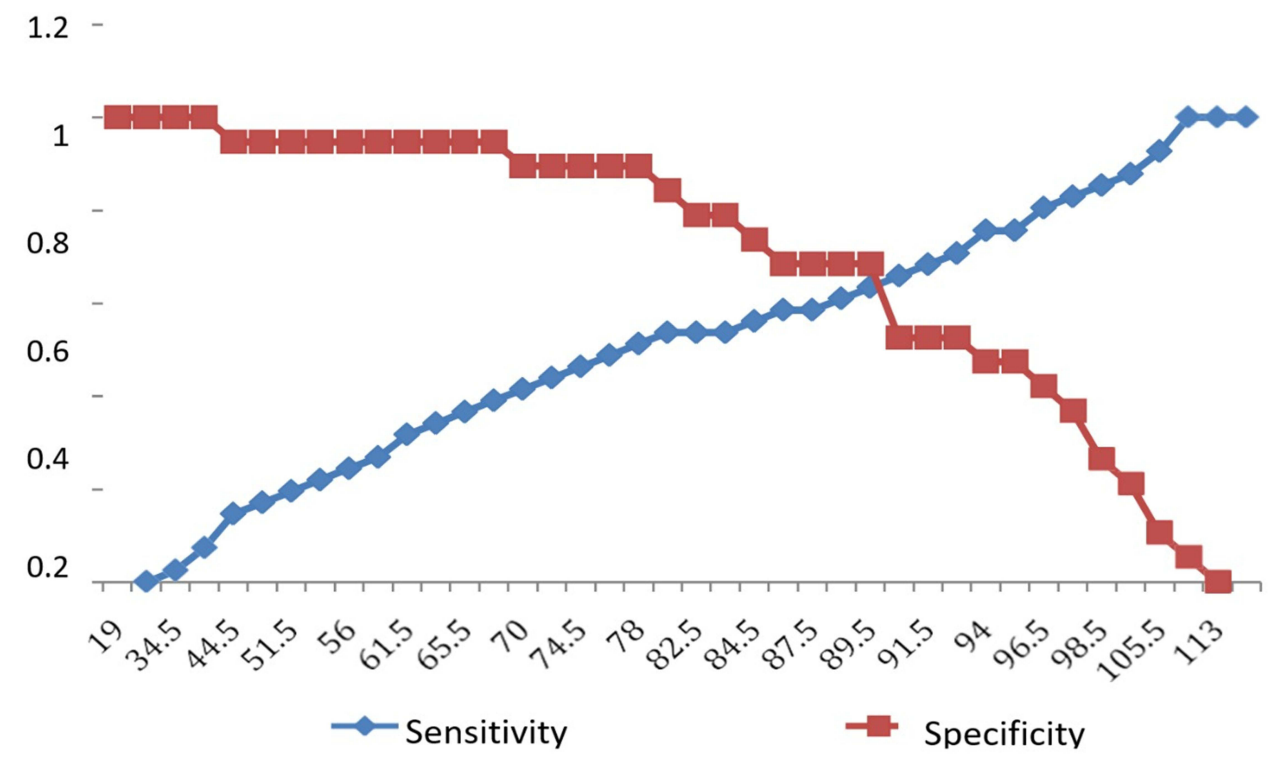

Figure 2 Cutoff point of transferrin saturation in distinguishing incidence of low bone density in adult patients with thalassemia. 
This study analyzed the correlation between bone mass density with transferrin saturation, ferritin serum, and Singh index. The result showed an inverse correlation between bone mass density and transferrin saturation with a value of $\mathrm{r}=-0.329$ and $\mathrm{p}=0.01$. On the other hand, there was no significant correlation between bone mass density and ferritin $(\mathrm{r}=-0.088 \mathrm{p}=0.504)$. This is the first study that obtained a significant correlation between the bone mass density and transferrin saturation in adult patients with thalassemia. Although the correlation was weak, it was statistically significant. Piga et $\mathrm{al}^{18}$ showed a correlation between nontransferrin-bound iron (NTBI) with transferrin saturation $(r=0.52 \mathrm{p}<0.001)$, but there was no correlation with serum ferritin. These findings might suggest that transferrin saturation can be used as a surrogate of bone mass density if the examination is not available.

These results also showed that there was no significant correlation between ferritin and bone density, which was similar to a previous study by Jensen et al. ${ }^{10}$ Ferritin is a protein that works as a storage of iron in the human body. Moreover, the levels are influenced by many factors besides iron overload, such as inflammatory reactions, liver damage, and malignancy. ${ }^{19,20}$ To overcome this issue, hs-CRP values were measured to exclude inflammatory conditions, while the average values of ferritin were used by examining 3 to 5 measurements in the last oneyear. Nonetheless, after excluding infection and averaging the serum ferritin levels, there was still no significant correlation between serum ferritin and bone mass density.

The Singh index is chosen since it can be obtained from plain femoral radiograph, which is available worldwide, even in remote healthcare facilities. Since bone mass density examination is not available throughout Indonesia, we want to determine if it can be used to detect low bone mass density. We found weak correlation between Singh Index and bone mass density $(r=0.273$, $p$-value $=0.038)$. This study provides preliminary data for further research to strengthen the evidence supporting the use of Singh index for detection of low bone mass density in patients with thalassemia.

With the discovery of the correlation between transferrin saturation with bone mass density, we determined the cut-off point of transferrin saturation to distinguish the incidence of low and normal bone density in adult patients with thalassemia. The Receiver Operator Curve (ROC) obtained a value of Area Under the Curve (AUC) 0.727 (95\% CI: 0.595-0.858), which showed that transferrin saturation has a good value to distinguish between low and normal bone density. Based on the ROC curve obtained, transferrin saturation values best cut-off point was $89.5 \%$.

The limitation of this study is the absence of hormonal assessment and its relation to bone mass density; therefore, it is recommended for future study. The clinical application of this study is to show that transferrin saturation can be used as a surrogate marker of low bone density in adult patients with thalassemia that has not pass through DXA examination.

\section{Conclusion}

The percentage of low bone mass density in patients with thalassemia major and intermedia adults in Cipto Mangunkusumo Hospital was $68 \%$. Based on the results, there was a significant inverse correlation between bone mass density with transferrin saturation, while there was no correlation between bone mass density with ferritin saturation in adult thalassemia major and intermedia patients. There was weak correlation between the Singh index and femoral bone density. Transferrin saturation cutoff point value to distinguish between the incidence of low and normal bone density is $89.5 \%$.

\section{Data Sharing Statement}

Additional data can be requested by contacting the corresponding author on the email address provided.

\section{Acknowledgments}

This paper is based on thesis: "Correlation of Transferrin Saturation and Serum Ferritin with Bone Mass Density in Adult Transfusion Dependent Beta-Thalassemia Patients" by Tenggara and Atmakusuma in $2016 .^{21}$

\section{Funding}

This paper is self-funded.

\section{Disclosure}

The authors report no conflicts of interest in this work.

\section{References}

1. Rachmilewtiz E, Giardina P. How I treat thalassemia. Blood. 2011;118:3479-3488. doi:10.1182/blood-2010-08-300335

2. Thein SL. Pathophysiology of $\beta$-thalassemia-A guide to molecular therapies. Am Soc Hematol Educ Prog. 2005;2005:31-37. doi:10.1182/asheducation-2005.1.31

3. Mahachoklertwattana P, Sirikulchayanonta V, Chuansumrit A, et al. Bone histomorphometry in children and adolescents with beta-thalassemia disease: iron-associated focal osteomalacia. J Clin Endocrinol Metab. 2003;88:3966-3972. doi:10.1210/jc.2002-021548 
4. Kremastinos DT, Farmakis D. Iron overload cardiomyopathy in clinical practice. Circ. 2011;124:2253-2263. doi:10.1161/CIRCULATI ONAHA.111.050773

5. Hershko C. Pathogenesis and management of iron toxicity in thalassemia. Ann NY Acad Sci. 2010;1202:1-9. doi:10.1111/j.17496632.2010.05544.x

6. Papanikolaou G, Pantopoulos K. Pantopoulos iron metabolism and toxicity. App Toxicol Pharmacol. 2005;202:199-211. doi:10.1016/j. taap.2004.06.021

7. Voskaridou E, Tepos E. New insights into the pathophysiology and management of osteoporosis in Patients with beta-thalassemia. Br J Haematol. 2004;127:127-139. doi:10.1111/j.1365-2141.2004.05143.x

8. Pootrakul P, Hungsprenges S, Fucharoen S, et al. Relation between erythropoiesis and bone metabolism in thalassemia. $N$ Eng $J$ Med. 1981;304:1470-1473. doi:10.1056/NEJM198106113042406

9. Michelson J, Cohen A. Incidence and treatment of fractures in thalassemia. J Orthop Trauma. 1988;2:29-32. doi:10.1097/00005131198802000-00007

10. Jensen CE, Tuck BC, Agnew JE, et al. High prevalence of low bone mass in thalassemia major. Brit J Haematol. 1998;103:911-915. doi:10.1046/j.1365-2141.1998.01108.x

11. Vichinsky EP. The morbidity of bone disease in thalassemia. Ann NY Acad Sci. 1998;850:344-348. doi:10.1111/j.1749-6632.1998.tb10491.x

12. Abdollah SA, Bekheirnia MR, Kamgar M, et al. Metabolic and endocrinologic complications in beta-thalassemia major: a multicenter study in Tehran. BMC Endocr Disord. 2003;3:1-6.

13. Hashemieh M, Azarkeivan A, Radfar M, et al. Prevalence of osteoporosis among thalassemia patients with thalassemia clinic from Zafar adult, Iran. J Iran Blood Cancer. 2014;6:143-148.
14. Tirtarahardja G, Setyohadi B, Weynand LS, Zhou Q. Bone mineral density reference values for Indonesian men and women; 2006. Available from: https://www.researchgate.net/publication/ 297712953. Accessed March 10, 2016.

15. DiStefano M, Chiabotto P, Roggia C, Garofalo F, Laura R. Bone mass and metabolism in thalassemic children and adolescents treated with different iron-chelating drugs. $J$ Bone Miner Metab. 2004;22:53-57. doi:10.1007/s00774-003-0449-z

16. Grampp S, Genant HK, Mathur A, et al. Comparisons of noninvasive bone mineral measurements in assessing age-related loss, fracture discrimination, and diagnostic classification. $J$ Bone Miner Res. 1997;12:697-711. doi:10.1359/jbmr.1997.12.5.697

17. Merchant R, Udani A, Puri V, et al. Evaluation of osteopathy in thalassemia by bone mineral densitometry and biochemical indices. Indian J Pediatr. 2010;77:987-991. doi:10.1007/s12098-010-0158-2

18. Piga A, Longo F, Duca L, et al. High nontransferrin bound iron levels and heart disease in thalassemia major. Am J Hematol. 2009;84:2933. doi:10.1002/ajh.21317

19. Ogilvie C, Fitzsimons K, Fitzsimons E. Serum ferritin values in primary care: high values are overlooked. $J$ Clin Pathol. 2010;63:1124-1126. doi:10.1136/jcp.2010.083188

20. Li CK, Chik KW, Lam CWK, et al. Liver disease in transfusiondependent thalassemia major. Arch Dis Child. 2002;86:344-347. doi:10.1136/adc.86.5.344

21. Tenggara JB, Atmakusuma TD, Yulisa ND, Abdullah M. Correlation of transferrin saturation and serum ferritin with bone mass density in adult transfusion dependent beta thalassemia patients. Thesis. 2016.
Journal of Blood Medicine

\section{Publish your work in this journal}

The Journal of Blood Medicine is an international, peer-reviewed, open access, online journal publishing laboratory, experimental and clinical aspects of all aspect pertaining to blood based medicine including but not limited to: Transfusion Medicine; Blood collection, Donor issues, Transmittable diseases, and Blood banking logistics; Immunohematology; Artificial and alternative blood based

\section{Dovepress}

therapeutics; Hematology; Biotechnology/nanotechnology of blood related medicine; Legal aspects of blood medicine; Historical perspectives. The manuscript management system is completely online and includes a very quick and fair peer-review system. Visit http://www.dovepress.com/testimonials.php to read real quotes from published authors. 